\title{
RESEARCH
}

\section{Comparison of elapsed times from breast cancer detection to first adjuvant therapy in Nova Scotia in 1999/2000 and 2003/04}

\author{
Daniel Rayson, Nathalie Saint-Jacques, Tallal Younis, Jason Meadows, Ron Dewar
}

\section{Abstract}

Background: Waiting times for cancer care continue to be an important issue for Canadians. We evaluated 2 cohorts of breast cancer patients to compare changes in elapsed times to care, to determine the proportion of patients who received their postoperative oncology consultation within the recommended time and to examine elapsed times between date of surgery and start of first adjuvant therapy.

Methods: We conducted a retrospective chart review of all women with surgically treated breast cancer who were referred to a provincial cancer centre for adjuvant therapy. The first cohort comprised women referred between Sept. 1, 1999, and Sept. 1, $2000(n=342)$, and the second cohort comprised women referred between Sept. 1, 2003, and Sept. $1,2004(n=295)$. A general linear model with a stepwise selection was used to identify dominant factors that influenced elapsed times; covariates included cohort period, age at diagnosis, place of residence, disease stage, type of surgery, type of adjuvant therapy, distance to cancer centre, median household income and mean education level.

Results: The overall median time from disease detection to the start of first adjuvant therapy for the combined cohorts was 96 days (quartiles 76,122 ); this interval was longer for patients in the second cohort ( $90 \mathrm{v.} 102$ days, $p<0.001$ ). For the combined cohorts, significantly more patients saw a radiation oncologist within the recommended time from date of surgery than did patients referred to a medical oncologist ( $82.7 \%$ v. $51.7 \% ; p<0.001)$. Patients who received adjuvant radiation therapy as their first adjuvant treatment waited longer from the date of definitive surgery to the start of treatment than did patients who received chemotherapy or hormonal treatment (77 v. 48 or 42 days; $p<0.001$ ).

Interpretation: The median elapsed time from the detection of breast cancer to the start of first adjuvant therapy was longer in the second cohort (referred in 2003/04) than in the first cohort (referred in 1999/2000). The proportion of patients whose first oncology consultation was within the recommended timeframe varied significantly according to type of oncology specialist, favouring radiation oncology. Despite this difference in access, patients whose first adjuvant therapy was systemic therapy experienced significantly shorter elapsed times from surgery to the start of adjuvant therapy than did patients whose first adjuvant therapy was radiation therapy.

$C M A J 2007 ; 176(3): 327-32$

$\mathrm{W}$ aiting times for cancer care continue to be an important issue for Canadians. ${ }^{1-5}$ Most analyses examining this question lack data clearly documenting time intervals to care. Data are even more limited for analyses of time between and over different components of care for a single diagnosis. ${ }^{6}$

Comprehensive care for early stage breast cancer is characterized by a sequence of events along a care path extending from first clinical or mammographic suspicion of disease to the completion of all adjuvant therapies and follow-up. Compared with many other malignant diseases, the steps for the evaluation and treatment of early stage breast cancer are relatively standardized for the vast majority of patients for whom surgery is potentially curative and adjuvant therapies are commonly recommended.

We previously published data on elapsed times from breast cancer detection to start of first adjuvant therapy in Nova Scotia for 1999/2000. ${ }^{7}$ We examined a second patient cohort examining the same sequence of events over a subsequent I2-month period with the following objectives: (a) to document and compare elapsed times from first clinical or mammographic detection of breast cancer to start of first adjuvant therapy for surgically resected breast cancer over 2 periods (1999/2000 and 2003/04); (b) to examine elapsed times between the date of last definitive surgery and the first consultation with an oncologist to assess the proportion of women who received their consultation within the time recommended in provincial guidelines and to assess the impact of covariates on this elapsed time; and (c) to examine elapsed times between the date of last definitive surgery and day I of the first adjuvant therapy and to assess the impact of the type of first adjuvant treatment on this elapsed time. 


\section{Methods}

Women in the first cohort were those who had an initial breast abnormality detected clinically or mammographically by Sept. I, I999, and were referred to I of 2 cancer centres in Nova Scotia (either the Nova Scotia Cancer Centre in Halifax or the Cape Breton Cancer Centre in Sydney) by Sept. I, 2000. The second cohort met the same eligibility criteria but had their initial breast abnormality detected by Sept. I, 2003, and were referred to one of the cancer centres by Sept. I, 2004 .

All patients were women who lived in Nova Scotia and had newly diagnosed invasive breast cancer for which potentially curative surgery was undertaken. Women with only in situ disease (ductal or lobular), synchronous cancers or metastatic disease were excluded (Fig. I).

Data were abstracted by 2 of us (J.M. and D.R.) from original radiologic, surgical and pathological reports contained in the patient charts at the cancer centres. In addition, data were obtained by 3 of us (J.M., R.D. and N.S.-J.) from the Oncology Patient Information System (OPIS), a computerized register of all cancer diagnoses and deaths in Nova Scotia since 1964 and composed of the Nova Scotia Cancer Registry and the provincial cancer centre treatment databases. The only missing values were those for an event not experienced by an individual patient.

Data quality in OPIS is ensured through online system edits, routine edits and periodic chart audits. All disease coding and demographic information are abstracted by trained health record professionals. We conducted weekly reviews of abstracted information to ensure the accurate capture of information, and data from 60 randomly selected charts were re-abstracted for validation purposes.

Data collected included patient age at time of referral, place of residence (Halifax and Hants counties, Cape Breton Island, or elsewhere in the province), disease stage at pathological review of surgical specimen, type of first adjuvant therapy received (chemotherapy, hormonal therapy or radiation therapy), as well as distance to the nearest cancer centre, median household income and mean education levels in area of residence (derived from Nova Scotia aggregate census data).

Dates abstracted included first clinical or mammographic evidence of breast cancer, first pathological confirmation of invasive disease, final definitive surgery, receipt of referral to one of the provincial cancer centres for consideration of adjuvant systemic or radiation therapy, patient contact by the cancer centre referral office or physician's office, first appointment with a medical or radiation oncologist and initiation of first adjuvant therapy (hormonal, chemotherapy or radiation).

For disease detection we used the date of first abnormal radiographic evidence (through mammography or ultrasonography) or, if specified in the chart, clinical disease detection in cases with a palpable abnormality. If there was uncertainty regarding the date of disease detection, the $15^{\text {th }}$ of the month preceding the date of pathological confirmation was used. In cases of discrepancy between the cancer centre chart and the OPIS data, we used dates from the cancer cen- tre chart, because it contained all original-source documentation and is the most complete source of information for all referred patients. For patients who received hormonal therapy as their first adjuvant therapy, the date of the first oncology consultation was used if the first day of adjuvant therapy was missing.

Elapsed times were calculated for 6 isolated care intervals (disease detection to biopsy, biopsy to surgery, surgery to referral, referral to patient contact, patient contact to consultation, and consultation to first adjuvant therapy), 4 composite intervals (disease detection to referral, referral to first adjuvant therapy, surgery to consultation, and surgery to first adjuvant therapy) and I overall interval (disease detection to first adjuvant therapy), as outlined in Fig. 2. All care intervals were calculated in calendar days, and only patients who actually experienced both events within an interval were included in the analysis of the defined interval.

A general linear model with a stepwise selection of significant covariates $(p<0.05)$ was used to identify the dominant factors influencing elapsed times. These covariates included cohort period, age at diagnosis, place of residence, disease stage, type of definitive surgery (modified radical mastectomy or breast conservation), first adjuvant therapy (chemotherapy, hormonal therapy or radiation therapy), distance to cancer centre, median household income and mean education level.

Days were logarithmically transformed (In[days $+\mathrm{I}]$ ) to better meet the assumption of data normality. ${ }^{8}$ Geometric

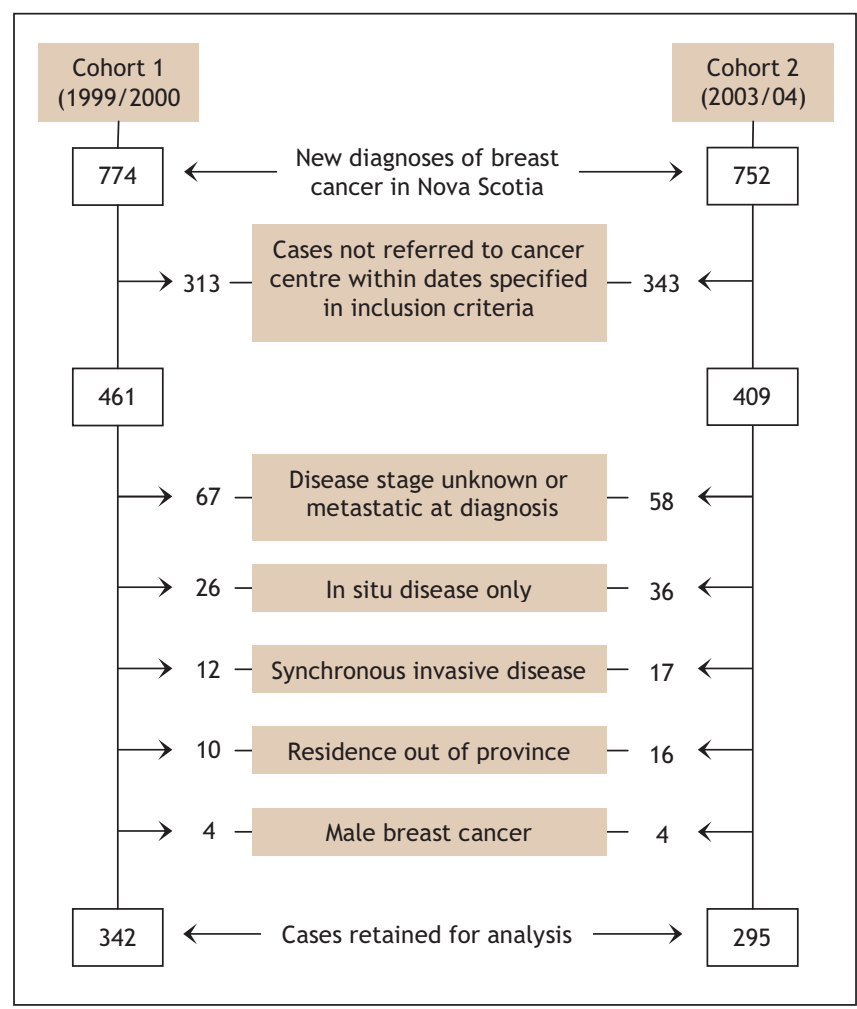

Fig 1: Case selection and exclusion criteria for 2 cohorts of patients with newly diagnosed breast cancer referred to a Nova Scotia cancer centre. Some cases may have been excluded for more than one reason. 
mean elapsed times and their $95 \%$ confidence intervals were estimated from the fully adjusted model, which accounted for the combined influence of factors and their first-order interaction with the cohort period. A Tukey a posteriori test was used for pairwise comparisons on the geometric mean elapsed times to determine significant differences among each covariate subgroup (treatment level). Data quality controls and analyses were performed using SAS software (version 9.I) and R software (version 2.I.o).

\section{Results}

A total of 637 women met the inclusion criteria and were included in the study: 342 were in the first cohort and 295 in the second (Fig. I). The proportion of patients residing on Cape Breton Island compared with elsewhere in the province increased significantly in the second cohort $(23.4 \% \mathrm{v}$. I4. $3 \%$; $p=0.013$ ) (Table I). As well, the proportion of patients who underwent breast conservation surgery compared with modified radical mastectomy was higher in the second cohort than in the first, although this difference did not reach statistical significance ( $48.3 \%$ v. $40.4 \% ; p>0.05$ ).

Fig. 2 shows the median elapsed times (and quartiles) for all of the intervals assessed. For the combined cohorts, a median of 96 days elapsed between disease detection to receipt of first adjuvant therapy (quartiles 76, I 22 days $[25 \%$ of patients experienced an elapsed time of more than 122 days between disease detection and start of first adjuvant therapy]). Patients in the first cohort experienced shorter mean elapsed times over this interval compared with those in the later cohort (period effect: $90 \mathrm{v}$. I02 days; $p<0.00 \mathrm{I})$. The elapsed times were significantly longer in the later cohort than in the first cohort for 3 of the 6 care intervals: biopsy to surgery (2I v. I7; $p=0.005$ ), surgery to referral ( $16 \mathrm{v} . \mathrm{I} 4 ; p=0.034$ ) and consultation to first adjuvant therapy (I2 v. 8; $p<0.001$ ) (Fig. 2).

Factors influencing the time from definitive surgery to consultation with an oncologist are shown in Table 2. No significant difference in elapsed time for this interval was observed between the 2 cohort periods. Patients residing on Cape Breton Island experienced a shorter elapsed time for this interval than did patients residing in central Nova Scotia (Halifax and Hants counties) or elsewhere in the province, although this difference was not statistically significant. The elapsed time from surgery to consultation was significantly influenced by age at diagnosis, disease stage and type of surgery.

In Nova Scotia, provincial guidelines recommend that most patients with surgically treated breast cancer have their first consultation with an oncologist (medical or radiation) within 6 weeks after definitive surgery. For patients at lowest risk of recurrence, those with in situ disease only and those with a tumour less than $\mathrm{I} \mathrm{cm}$ in size and negative axillary lymph nodes, an 8-week interval is often used.

For the combined cohort, $55.3 \%$ of the patients had their first oncology consultation within the recommended 6-week timeframe (Table 3). For the patients for whom the 8-week timeframe was judged acceptable, $95.6 \%$ were seen within this recommended time.

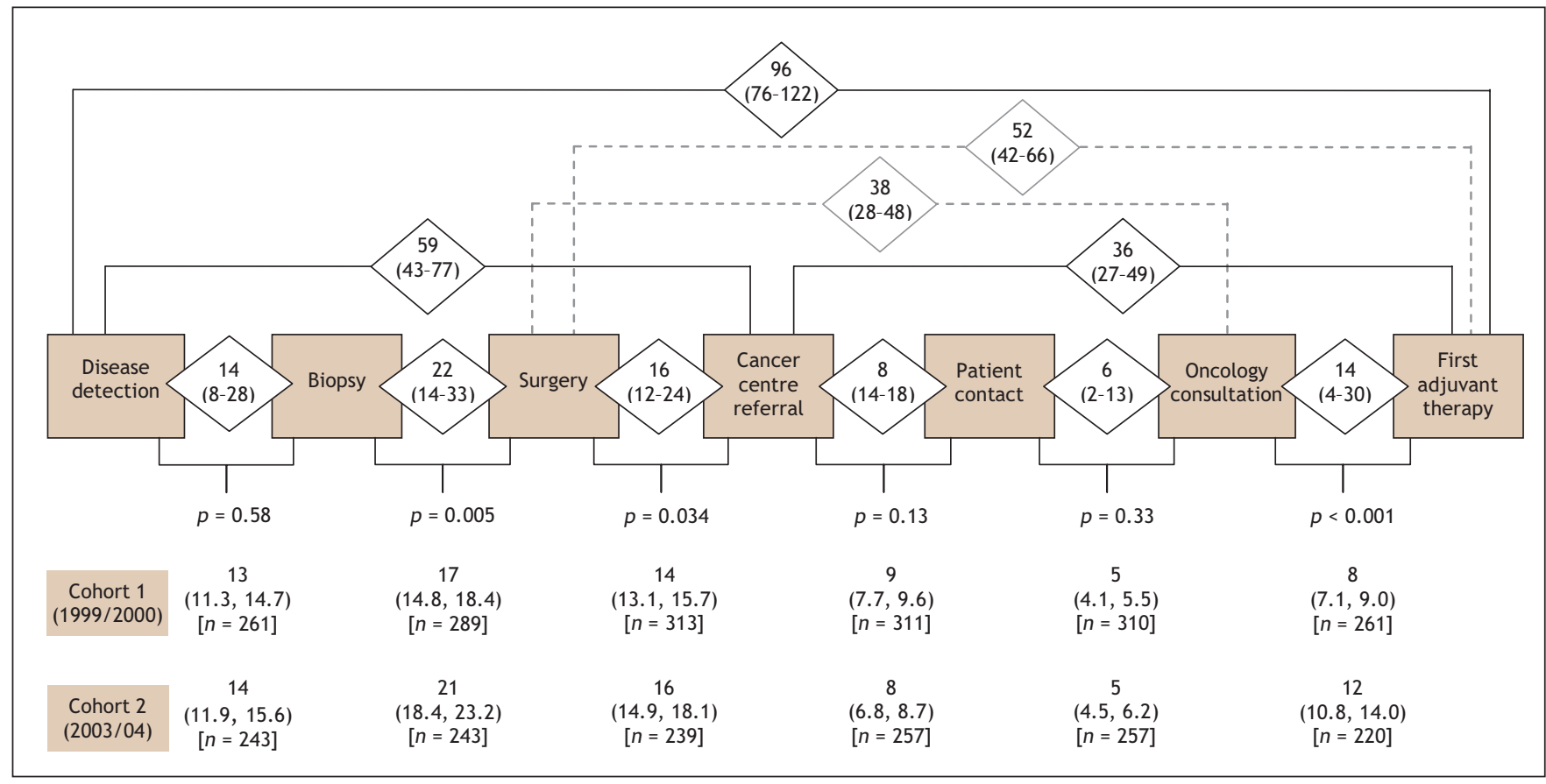

Fig. 2: Elapsed times between care intervals for women with newly diagnosed invasive breast cancer referred to a Nova Scotia cancer centre between Sept. 1, 1999, and Sept. 1, 2000 (cohort 1) and between Sept. 1, 2003, and Sept. 1, 2004 (cohort 2). Median number of days (unadjusted for cofactors) with first and third quartiles (cohorts combined) are shown in diamonds. Geometric mean number of days (adjusted for cofactors) with $95 \%$ confidence intervals (cohort comparison) are shown below each care interval. The significance in the differences in elapsed days observed between cohort periods is indicated by $p$ values. 
The proportion of women seen within the recommended timeframes varied by type of oncology specialist first consulted (radiation v. medical oncologist). Radiation oncologists saw a significantly greater proportion of patients within the recommended timeframes than did medical oncologists (cohorts combined; $82.7 \%$ v. $51.7 \%$; $p<0.001$ ). The proportion of patients seen by a radiation oncologist within the recommended timeframes increased significantly over time, from $76.2 \%$ in the first cohort to $87.9 \%$ in the second cohort ( $p=0.015)$. The proportion of medical oncology consultations performed within these timeframes decreased over time, although not significantly $(54.7 \%$ and $46.5 \%$ respectively; $p=0.14$ ).

Despite the different elapsed times from surgery to consultation by type of oncology specialist for the combined cohorts, patients who received radiation therapy as their first adjuvant therapy experienced significantly longer elapsed times from surgery to first adjuvant therapy than did patients who received either chemotherapy or hormonal therapy as their first adjuvant treatment (77 v. 48 or 42 days respectively; $p<0.00 I)$.

\section{Interpretation}

The overall median time from the detection of breast cancer to the start of first adjuvant therapy for the combined cohorts

Table 1: Characteristics of women with newly diagnosed invasive breast cancer in cohort 1 (referred to cancer centre in 1999/2000) and cohort 2 (referred to cancer centre in 2003/04)

\begin{tabular}{lrrr}
\hline & \multicolumn{2}{c}{ No. (\%) of women } & \\
\cline { 2 - 3 } Characteristic & Cohort 1 & Cohort 2 & p value* \\
\hline Place of residence & & & 0.013 \\
\hline $\begin{array}{l}\text { Halifax and Hants } \\
\text { counties (central) }\end{array}$ & $163(47.7)$ & $124(42.0)$ & \\
\hline Cape Breton Island & $49(14.3)$ & $69(23.4)$ & \\
Elsewhere in province & $130(38.0)$ & $102(34.6)$ & \\
\hline Age at diagnosis, yr & & & 0.74 \\
\hline$\leq 49$ & $95(27.8)$ & $74(25.1)$ & \\
\hline $50-69$ & $176(51.5)$ & $157(53.2)$ & \\
\hline$\geq 70$ & $71(20.8)$ & $64(21.7)$ & \\
\hline Disease stage & & & 0.76 \\
\hline I & $152(44.4)$ & $124(42.0)$ & \\
\hline II & $147(43.0)$ & $129(43.7)$ & \\
\hline III & $43(12.6)$ & $42(14.2)$ & \\
\hline Type of surgery & & & 0.06 \\
\hline Lumpectomy & $131(40.4)$ & $127(48.3)$ & \\
\hline Mastectomy & $193(59.6)$ & $136(51.7)$ & \\
\hline First adjuvant therapy & & & 0.33 \\
\hline Hormonal therapy & $63(21.2)$ & $64(26.1)$ & \\
\hline Chemotherapy & $157(52.9)$ & $116(47.4)$ & \\
\hline Radiotherapy & $77(25.9)$ & $65(26.5)$ & \\
\hline & & & \\
\hline
\end{tabular}

${ }^{*} \chi^{2}$ test for difference between cohorts. was 96 days (quartiles 79, I22), with a longer interval observed in the later cohort ( $90 \mathrm{v}$. I02 days; $p<0.00 \mathrm{I}$ ). The proportion of patients who had their first oncology consultation within the recommended timeframe varied significantly according to type of oncology specialist. Fewer patients saw a medical oncologist within the recommended timeframe compared with patients who saw a radiation oncologist $(5 \mathrm{I} .7 \%$ v. $82.7 \%$ respectively; $p<0.00 \mathrm{I})$. Despite this difference in access favouring radiation oncology, patients whose first adjuvant therapy was systemic therapy (chemotherapy, hormonal therapy) experienced significantly shorter elapsed times to the start of therapy after surgery than did patients whose first adjuvant therapy was radiation therapy (48 or 42 v. 77 days respectively; $p<0.001$ ).

The interval between date of definitive surgery and consul-

Table 2: Effect of factors on elapsed time from definitive surgery to consultation with an oncologist

\begin{tabular}{|c|c|c|c|}
\hline Factor & $\begin{array}{l}\text { No. of } \\
\text { patients }\end{array}$ & $\begin{array}{l}\text { No. of days, } \\
\text { adjusted geometric } \\
\text { mean }(95 \% \mathrm{Cl})^{*}\end{array}$ & $p$ value \\
\hline Cohort period & & & 0.99 \\
\hline $1999 / 2000$ & 305 & $36(34.0-38.1)$ & \\
\hline $2003 / 04$ & 246 & $36(33.9-38.5)$ & \\
\hline Place of residence & & & $>0.05$ \\
\hline $\begin{array}{l}\text { Halifax and Hants } \\
\text { counties }\end{array}$ & 248 & $38_{a}(35.7-40.5)$ & \\
\hline Cape Breton Island & 102 & $33_{a}(29.9-36.5)$ & \\
\hline $\begin{array}{l}\text { Elsewhere in } \\
\text { province }\end{array}$ & 201 & $35_{a}(32.9-37.8)$ & \\
\hline Age at diagnosis, yr & & & 0.004 \\
\hline$\leq 49$ & 139 & $33_{a}(30.2-35.7)$ & \\
\hline $50-69$ & 295 & $36 \mathrm{a}(34.0-38.1)$ & \\
\hline$\geq 70$ & 117 & $40_{b}(37.0-44.3)$ & \\
\hline Disease stage & & & $<0.001$ \\
\hline I & 238 & $37_{a}(34.6-39.4)$ & \\
\hline II & 240 & $38_{a}(35.9-40.8)$ & \\
\hline III & 73 & $27_{\mathrm{b}}(24.2-30.7)$ & \\
\hline Type of surgery & & & $<0.001$ \\
\hline Lumpectomy & 246 & $32(29.6-33.7)$ & \\
\hline Mastectomy & 305 & $40(37.9-42.5)$ & \\
\hline $\begin{array}{l}\text { Type of surgery by } \\
\text { cohort period }\end{array}$ & & & 0.008 \\
\hline \multicolumn{4}{|l|}{ Lumpectomy } \\
\hline $1999 / 2000$ & 127 & $33(30.6-36.6)$ & \\
\hline $2003 / 04$ & 122 & $29(26.8-32.2)$ & \\
\hline \multicolumn{4}{|l|}{ Mastectomy } \\
\hline $1999 / 2000$ & 179 & $38(35.4-41.1)$ & \\
\hline $2003 / 04$ & 129 & $43(39.0-46.7)$ & \\
\hline
\end{tabular}

Note: $\mathrm{Cl}=$ confidence interval.

*Statistically significant pairwise comparisons, resulting from analysis of variance a posteriori Tukey tests, are annotated with a different subscript. For example, In the category of age at diagnosis, patients 70 years and older experienced significantly longer elapsed times for this interval than did either patients aged 49 and less or those aged 50-69. 
tation with an oncologist has been the subject of increasing interest. It is one indicator of quality of cancer care that is often used as a measure of system efficiency, communication, capacity and resource utilization..$^{9-11}$ A number of provinces use this interval as a major or sole measure of cancer care wait times on public Web sites. ${ }^{12-14}$

For patients, this period is one of tremendous anxiety, and some data suggest that prolonged wait times from definitive surgery to initiation of adjuvant systemic therapy may be associated with negative outcomes, particularly for patients at highest risk of metastatic disease..$^{15-17}$

Recent evidence suggests that adjuvant chemotherapy for breast cancer is equally effective up to I2 weeks after definitive surgery but that relapse-free and overall survival may be compromised with delays greater than $\mathrm{I} 2$ weeks. ${ }^{18}$ The mean time from definitive surgery to initiation of adjuvant chemotherapy observed in our cohorts was 48 days ( $95 \%$ confidence interval 45-5I days), which suggests that, despite long waits to see a medical oncologist, the vast majority of patients received chemotherapy within a clinically acceptable timeframe.

We observed shorter times from surgery to chemotherapy or hormonal therapy than from surgery to radiation therapy. This difference may have been due to the fact that a recommendation to proceed to systemic chemotherapy results in treatment initiation within a few days of the consultation, as compared with a typical interval of a number of weeks before initiation of radiation therapy. As well, the decision to proceed to radiation therapy may be delayed until a final decision

Table 3: Patients whose consultation with an oncologist after definitive surgery was within the provincially recommended timeframe*

$\%$ of women seen within recommended timeframe (no. eligible) $\dagger$

\begin{tabular}{|c|c|c|c|}
\hline Variable & 6 weeks & 8 weeks & Total \\
\hline \multicolumn{4}{|l|}{ First oncology consultation } \\
\hline Cohort combined & $55.3(438)$ & $95.6(135)$ & $64.8(573)$ \\
\hline $1999 / 2000$ & $54.8(261)$ & $96.4 \quad(56)$ & $62.1(317)$ \\
\hline $2003 / 04$ & $55.9(177)$ & 94.9 (79) & $68.0(256)$ \\
\hline Period effect $\chi^{2}$ ( $p$ value) & $0.06(0.81)$ & $0.17(0.68)$ & $2.1(0.15)$ \\
\hline \multicolumn{4}{|l|}{ Medical oncologist } \\
\hline Cohort combined & $47.8(322)$ & $90.6 \quad(32)$ & $51.7(354)$ \\
\hline $1999 / 2000$ & $50.7(203)$ & $90.9 \quad(22)$ & $54.7(225)$ \\
\hline $2003 / 04$ & 42.9 (119) & $90.0 \quad(10)$ & 46.5 (129) \\
\hline Period effect $\chi^{2}$ ( $p$ value) & $1.9(0.17)$ & $0.01(0.94)$ & $2.2(0.14)$ \\
\hline \multicolumn{4}{|l|}{ Radiation oncologist } \\
\hline Cohort combined & $71.1(135)$ & $96.5(114)$ & $82.7(249)$ \\
\hline $1999 / 2000$ & $63.2(68)$ & $97.6 \quad(41)$ & $76.2(109)$ \\
\hline $2003 / 04$ & $79.1 \quad(67)$ & $95.9 \quad(73)$ & $87.9(140)$ \\
\hline Period effect $\chi^{2}$ ( $p$ value) & $4.1(0.042)$ & $0.22(0.64)$ & $5.9(0.015)$ \\
\hline
\end{tabular}

${ }^{*}$ Consensus-based provincial guidelines regarding time to first oncology consultation from date of final definitive surgery recommend that most patients be seen within 6 weeks after surgery. An 8-week timeframe is considered acceptable for women with ductal carcinoma in situ only (excluded in our study) or patients whose tumour size is $1 \mathrm{~cm}$ or smaller.

†Except where stated otherwise. is made regarding whether or not chemotherapy will be recommended. As such, longer elapsed times to a medical oncology consultation may significantly influence the timeliness of radiation therapy administration. This illustrates the importance of examining the interaction of elapsed times among different subspecialties involved with the sequential care of a disease, to more accurately understand elapsed times to care.

As a further example, investigators in the United Kingdom have found that waiting times from referral by a general practitioner to first hospital appointment have improved since the introduction of government targets highlighting this interval. However, times from first hospital appointment to treatment initiation have increased, with the result of a minimal change in overall care timelines. ${ }^{19}$

Our study has several limitations. We analyzed only referred cases within a limited timeframe and, therefore, did not perform a population-based analysis of breast cancer care times nor a complete analysis of care times over the entire interval that could have been potentially assessed (1999-2004). We did not assess variables that may have influenced overall care times, including number of staging investigations, postoperative complication rates, and missed or cancelled appointments. We excluded women with only in situ or metastatic disease and men with breast cancer; therefore, our data are not applicable to these patient populations. As well, we assessed the elapsed time to first adjuvant therapy only and therefore incompletely described the elapsed times for women undergoing a sequence of adjuvant therapies. Despite these limitations, our data remain consistent with those from our previous work and render our analysis of care times more robust for women meeting the inclusion criteria. As well, our methodology is one that could be easily reproduced in other provincial jurisdictions, which may enable generalization of the results and improve communication and transparency regarding wait times for breast cancer care.

Although often seen as a measure of cancer centre efficiency and resource allocation, the interval from date of definitive surgery to first oncology consultation or to initiation of first adjuvant therapy may be influenced by a series of factors that cannot be reflected in unidimensional data and may lead to false assumptions regarding elements contributing to elapsed times within the cancer system. For patients concerned about disease recurrence and timely initiation of adjuvant therapy, publicly available data regarding wait times to consultation with a medical or radiation oncologist may inappropriately heighten concern for those waiting to see a medical oncologist and falsely reassure those waiting to see a radiation oncologist. We suggest that examination of timelines over the entire continuum of breast cancer 
care may provide a more realistic picture of the burden of waiting times as well as improve our understanding of the interdependence of care segments on access and outcomes. Provincial Web sites reporting unidimensional wait times for single care events along an obligatory continuum may falsely represent the burden of waiting and provoke both unnecessary anxiety and unrealistic comfort to those in wait.

This article has been peer reviewed.

From the Faculty of Medicine, Dalhousie University, and the Cancer Care Program, Capital District Health Authority (Rayson, Younis, Meadows); and the Surveillance and Epidemiology Unit, Cancer Care Nova Scotia (SaintJacques, Dewar), Halifax, NS

Competing interests: None declared.

Contributors: Daniel Rayson conceived the project and designed the study and was intimately involved with all aspects of data acquisition, analysis and interpretation. Nathalie Saint-jacques contributed substantially to the data analysis and interpretation, and the design of the data programs. Tallal Younis provided important assistance in the analysis and interpretation of the data. Jason Meadows contributed substantially to data acquisition. Ron Dewar contributed substantially to the design of the data programs and to the acquisition and analysis of the data. All of the authors were involved in the drafting of the article and critical revisions to content and gave final approval for publication.

Acknowledgements: We thank the Nova Scotia Cancer Registry and the Nova Scotia Breast Cancer Site Team for their ongoing support. We also thank Sandra Bellefontaine for her expert administrative assistance.

\section{REFERENCES}

I. Wait Time Alliance for Timely Access to Health Care. It's about time! Achieving benchmarks and best practices in wait time management. Ottawa: Canadian Medical Association; 2005. Available: www.cma.ca/multimedia/CMA/Content_Images /Inside_cma/Media_Release/pdf/2005/wta-final.pdf (accessed 2006 Nov I7).

2. Savage C. Tracking system delays: long waits and no short answers. In: Cancer Advocacy Coalition of Canada. Report card 2004. Vol 7, Winter 2004/05. p. 4-9. Available: www.canceradvocacy.ca/reportcard/2004/cancer-advocacy-coalition-reportcard-2004.pdf (accessed 2006 Nov 17).

3. Spilchuk J. So you have cancer - Hurry up and wait. In: Cancer Advocacy Coalition of Canada. Report card 2005. Vol 8, Winter 2005/06. p. 6-7. Available: www
canceradvocacy.ca/reportcard/2005/REPORT_CARD_2005.pdf (accessed 2006 Nov I7).

4. Rayson D. Cancer care waits: time elapsed and time wasted. In: Cancer Advocacy Coalition of Canada. Report card 2005. Vol 8, Winter 2005/o6. p. 8-9. Available: www.canceradvocacy.ca/reportcard/2005/REPORT_CARD_2005.pdf (accessed 2006 Nov I7).

5. Savage C. Provincial wait times. In: Cancer Advocacy Coalition of Canada. Report card 2005. Vol 8, Winter 2005/06. p. I0-2. Available: www.canceradvocacy.ca/reportcard/2005/REPORT_CARD_2005.pdf (accessed 2006 Nov I7).

6. McIntosh T. The taming of the queue II: Colloquim report. March 3I-April I, 2005. Available: www.cprn.com/en/doc-download.cfm?id=I274\&pdf=37922_en .pdf (accessed 2006 Nov I7).

7. Rayson D, Chiasson R, Dewar R. Elapsed time from breast cancer detection to first adjuvant therapy in a Canadian province I999-2000. CMAJ 2004;170:957-6I.

8. Armitage P, Berry G. Statistical methods in medical research. 3 rd ed. Oxford (UK): Blackwell Science; I994.

9. Cancer Care Ontario. Cancer system quality index 2006. Available: www.cancercare.on.ca/qualityindex (accessed 2006 Nov 17)

Io. Health Canada. Wait times in Canada. Available: www.hc-sc.gc.ca/hcs-sss/qual /acces/wait-attente/index_e.html (accessed 2006 Nov I7).

II. Canadian Institutes of Health Research. CIHR releases research results to inform the development of benchmarks for wait times. Available: www.cihr-irsc.gc.ca/e 129903.html (accessed 2006 Nov 17).

I2. Cancer Care Ontario. Statistics \& research. Wait times. Available: www.cancercare .on.ca/index_statisticsandresearch.htm (accessed 2006 Nov I7).

13. Alberta Waitlist Registry. Waitlist frequently asked questions. Available: www.ahw.gov.ab.ca/waitlist/AWRInfoPage.jsp?pageID=9 (accessed 2006 Nov I7).

I4. Nova Scotia Department of Health Website. Nova Scotia wait times. Available: www.gov.ns.ca/heal/waittimes (accessed 2006 Nov I7).

15. Rayson D. Cancer care waits: time elapsed and time wasted. Cancer Care Advocacy Coalition report card Vol 8, Winter 2005/06. Available: www.canceradvocacy.ca /reportcard/2005/REPORT_CARD_2005.pdf (accessed 2006 Nov I7).

I6. Colleoni M, Bonetti M, Coates AS, et al. Early start of adjuvant chemotherapy may improve treatment outcome for premenopausal breast cancer patients with tumors not expressing estrogen receptors. The International Breast Cancer Study Group. J Clin Oncol 2000;18:584-90.

I7. Hershman DL, Wang X, McBride RB, et al. Delay of adjuvant chemotherapy initiation following breast cancer surgery among elderly women. Breast Cancer Res Treat 2006;99:313-21.

I8. Lohrisch C, Paltiel C, Gelmon K, et al. Impact on survival of time from definitive surgery to initiation of adjuvant chemotherapy for early-stage breast cancer. J Clin Oncol 2006;24:4888-94.

I9. Robinson D, Bell CM, Moller H, et al. Effect of the UK government's 2 week target on waiting times in women with breast cancer in southeast England. BrJ Cancer 2003;89:492-6.

Correspondence to: Dr. Daniel Rayson, Medical Oncology, Rm. 46o, Bethune Building, QEII Health Sciences Centre, ${ }_{2} 278$ Tower Rd., Halifax NS $\mathrm{B}_{3} \mathrm{H}_{2} \mathrm{Yg}$;

daniel.rayson@cdha.nshealth.ca

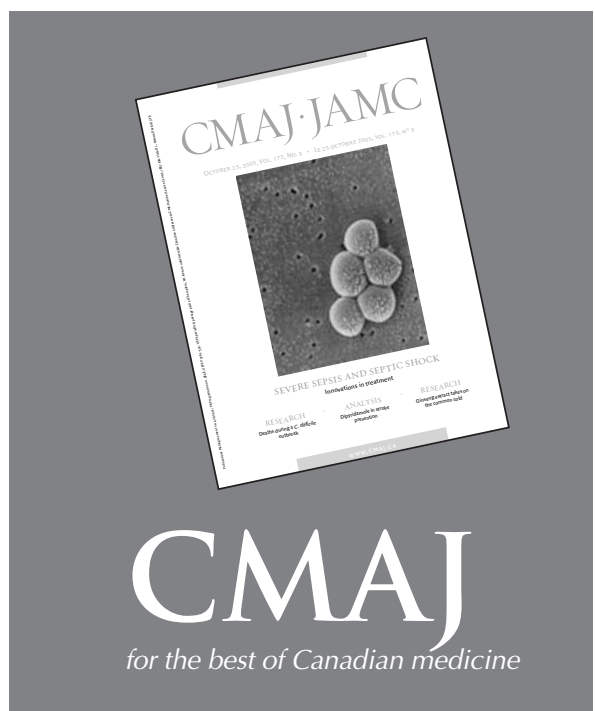

3000 volunteer reviewers from around the world are the foundation for CMAJ's thorough, criteriabased review process. The editorial staff includes scientific consultants with expertise in statistics, experimental design and epidemiology.

\section{CMA Member Service Centre tel $888855-2555$ or $613731-8610 \times 2307$ fax $613236-8864$ cmamsc@cma.ca}

